\section{WHICH ARTIFICIAL ODOURS DO PREMATURE NEWBORNS SMELL IN THEIR INCUBATOR? A FRENCH SURVEY OF HEALTHCAREI CLEANING PRODUCTS USE IN NICU}

\author{
P. Kuhn ${ }^{1,2}$, D. Astruc ${ }^{1}$, J. Messer ${ }^{1}$, L. Marlier ${ }^{2}$
}

${ }^{1}$ Médecine et Réanimation Néonatale - Service de Pédiatrie 2, Centre Hospitalier Universitaire de Strasbourg, 'Laboratoire d'Imagerie et de Neurosciences Cognitives FRE 3289, Centre National de la Recherche Scientifique - Université de Strasbourg, Strasbourg, France

Aims: To assess the healthcare products and cleaning solutions used in neonatology units throughout France and to evaluate the main determinants of the newborn's nosocomial olfactory environment.

Methods: An electronic questionnaire was sent to 99 neonatal units in France. Senior nurses and/or physicians described the nature and use of skincare products (e.g., umbilical cord and skin care products, skin disinfectants, adhesive removers), lubrications used for tube positioning, and disinfectants used to clean materials such as incubators or ultrasound probes, hand hygiene products (e.g., alcohol-based hand rubs, antimicrobial and plain soaps), and newborns' bath.

Results: We achieved a $49.5 \%$ (i.e., 49 of 99) response rate. Nine groups of products and 76 distinct commercial preparations were identified. Depending on their vulnerability, newborns infants were estimated to be exposed to nosocomial odours an average of 48 to 64 times daily, although higher levels were reported by some respondents. Use of the products varied among the medical centres. Newborns were most frequently exposed to the odour of hand hygiene products (aqueous alcoholic solutions, soaps) and skin antisepsis products.

Conclusions: This survey reveals that newborns are exposed to high levels of olfactory and trigeminal stimuli of nosocomial origin, thereby increasing the complexity of the newborn's olfactory environment. The impact of these stimuli on the short-term wellbeing and long-term development of newborns should be systematically evaluated. Although most of these products are necessary, a wise use of them is recommended. Consensus guidelines should be developed and promoted to regulate their use.

\section{THE DISPARATE PSYCHOLOGICAL SUPPORT AVAILABLE FOR PARENTS IN UK TERTIARY LEVEL NEONATAL UNITS}

\author{
J. Alam ${ }^{1}$, S. Ahlund ${ }^{1}$, N.K. Thalange ${ }^{2}$, P. Clarke ${ }^{1}$ \\ ${ }^{1}$ Neonatal Intensive Care Unit, Norfolk and \\ Norwich University Hospital, Norwich, UK, '2Jenny \\ Lind Children's Department, Norfolk and Norwich \\ University Hospital, Norwich, UK
}

Background and aims: Neonatal intensive care units (NICUs) are high stress environments for parents and staff. Mothers of very low birth weight infants have a high prevalence of post-traumatic stress disorder and anxiety at 2-3 years post delivery. Maternal anxiety in the NICU affects maternal-child interaction, and infant cognitive and socio-emotional development assessed at 24 months corrected age. We aimed to assess current psychological and social support available for parents in UK tertiarylevel NICUs.

Methods: Telephone survey of all tertiary-level UK NICUs in August 2009 to assess psychological and social support provided to parents.

Results: 49/49 (100\%) NICUs responded. Only 2 units $(4 \%)$ had clinical psychologists specifically dedicated to their NICUs; 14 units $(29 \%)$ had limited clinical psychologist cover; 13 units $(27 \%)$ offered formal psychological support to parents although not via a clinical psychologist; 20 (41\%) units reported that no formal psychological support was available for parents.

Only 19 (39\%) units had regular input from a social worker with dedicated responsibility for the NICU, whereas most NICUs $(30 / 49 ; 61 \%)$ had neither a dedicated social worker nor any regular meeting between nurses/doctors and a social worker to discuss the social needs of parents/families.

Conclusion: Our survey highlights the disparate current state of psychosocial support available for parents with babies on UK NICUs. Some NICUs offer parents excellent support, while many others offer no formal psychological or dedicated social support. Optimising parental psychosocial support has the potential to minimise adverse long-term consequences for infants and their families. 